\title{
BALAYAGE IN FOURIER TRANSFORMS: GENERAL RESULTS, PERTURBATION, AND BALAYAGE WITH SPARSE FREQUENCIES
}

BY

\author{
GEORGE S. SHAPIRO
}

\begin{abstract}
Let $\Lambda$ be a discrete subset of an LCA group and $E$ a compact subset of the dual group. Balayage is said to be possible for $(\Lambda, E)$ if the Fourier transform of each measure on $G$ is equal on $E$ to the Fourier transform of some measure supported by $\Lambda$. Following Beurling, we show that this condition is equivalent to the possibility of bounding certain functions with spectra in $E$ by their bounds on $\Lambda$. We derive consequences of this equivalence, among them a necessary condition on $\Lambda$ for balayage when $E$ is compact and open (a condition analogous to a density condition Beurling and Landau gave for balayage in Euclidean spaces).

We show that if balayage is possible for $(\Lambda, E)$ and if $\Lambda^{\prime}$ is close to $\Lambda$, then balayage is possible for $\left(\Lambda^{\prime}, E\right)$. Explicit bounds for the needed closeness in $R$ and $R^{n}$ are given.

Using these perturbation techniques, we give examples of perfect sets $E \subset R$ with the property that there are "arbitrarily sparse" sets $\Lambda$ with balayage possible for $(\Lambda, E)$.
\end{abstract}

1. Introduction and definitions. Beurling [2], [3] initiated the study of what he called "balayage of measures in Fourier transforms" in the case of Euclidean spaces and, more especially, the real line where he made profound use of complex variable techniques. As he was aware, many of his more elementary observations extend to general locally compact abelian groups.

Throughout, $G$ will denote a locally compact abelian group and $\Gamma$ the dual group of $G$, both written additively. We write $M(G)$ for the convolution algebra of regular, finite complex Borel measures on $G$. If $\Lambda \subset G$ is closed, $M(\Lambda)$ denotes the subspace of measures supported on $\Lambda$. If $\mu \in M(G)$ we write $\hat{\mu}$ for the (inverse) Fourier transform of $\mu$ so that

$$
\hat{\mu}(\gamma)=\int_{G}\langle\gamma, x\rangle d \mu(x) \text { for } \gamma \in \Gamma .
$$

Received by the editors August 20, 1975.

AMS (MOS) subject classifications (1970). Primary 43A25; Secondary 42A44, 42A48, 46E15.

Key words and phrases. Balayage in Fourier transforms, convolution, set of sampling, dual Banach spaces, surjective linear operator, density, uniformly discrete, relatively dense, compact open subgroup, sparse set, Dirichlet set, homogeneous perfect set. 
Definition 1.1. If $\Lambda \subset G$ is closed and $E \subset \Gamma$, then balayage is possible for the pair $(\Lambda, E)$ if for every $\alpha \in M(G)$ there is some $\mu \in M(\Lambda)$ with $\hat{\alpha}(\gamma)$ $=\hat{\mu}(\gamma)$ for all $\gamma \in E$.

We shall always assume that $\Lambda$ is discrete and $E$ is compact. Thus, for $\mu \in M(\Lambda)$ we have a series representation, $\hat{\mu}(\gamma)=\sum a_{\lambda}\langle\gamma, \lambda\rangle$, the sum being over $\Lambda$.

Some important examples of balayage are given in [2] where Beurling showed that if $\Lambda \subset R$ is uniformly discrete and $E \subset R$ is an interval then balayage is possible for $(\Lambda, E)$ if and only if the lower density $D_{-}(\Lambda)$ satisfies

$$
D_{-}(\Lambda)>(1 / 2 \pi) \text { meas }(E) \text {. }
$$

Here $D_{-}(\Lambda)=\lim _{r \rightarrow \infty} n(r) / r$ where $n(r)$ is the least number of points of $\Lambda$ contained in any interval of length $r$ and meas $(E)$ is the Lebesgue measure of $E$.

Landau [7], using $L^{2}$-methods, showed that, quite generally, (1) is necessary for balayage and that a similar result holds in $R^{n}$.

In $\$ 2$, we give a reformulation of the problem of balayage. The major result is Theorem 2.4 showing the equivalence of the problem of balayage with a certain sampling problem. This is inspired by a similar result of Beurling; however, we eliminate certain restrictions which he needed. We then apply this theorem in $\$ 3$ to give an analogue of the necessity of condition (1) for balayage in the case where $E$ is compact and open.

The remaining three sections explore and exploit perturbation results, that is, results which state that if balayage is possible for $(\Lambda, E)$ and if $\Lambda^{\prime}$ is "close" to $\Lambda$ (in senses to be made precise) then balayage is possible also for $\left(\Lambda^{\prime}, E\right)$.

$\$ 4$ contains a perturbation result for general LCA groups. In $\$ 5$, we give explicit bounds for the needed closeness of $\Lambda$ and $\Lambda^{\prime}$ in $R$ and $R^{n}$.

In $\S 6$, we study and construct perfect sets $E$ in $R$, called BAS sets, having the property that there are "arbitrarily sparse" sets $\Lambda$ with balayage possible for $(\Lambda, E)$. Here we use the techniques of $\$ 4$, an estimate from $\$ 5$ and a certain norm estimate. Using Theorem 6.4 we see that "thinness" per se does not imply the BAS property.

Some of our results are generalizations of Beurling's and much of our work was inspired by [2], which is, unfortunately, unpublished. Where relevant, we include remarks detailing the connections.

The original versions of some of the results here appeared in the author's dissertation [10]. The author wishes to thank his advisor, Professor L. Loomis for introducing him to the problem of balayage (and, indeed, to abstract analysis) and for his encouragement, and Professor G. Mackey for a helpful conversation. We also thank $\mathrm{H}$. Landau for making available his personal, handwritten copy of [2].

We now introduce some notations and conventions.

We regard $L^{1}(G)$ as a subset (and an ideal) of $M(G)$. All integrals $d x, d \gamma$, etc. are with respect to the relevant Haar measure. Mention of relations 
holding almost everywhere (a.e.) or locally a.e. refer to Haar measure.

The elements of $L^{\infty}(G)$ and $L^{1}(G)$ are equivalence classes of functions but will, as usual, be denoted $f, g, h$, etc. When we need to mention an element of such a class, we will denote it $f_{0}, g_{0}, h_{0}$, etc.

$\delta_{x}$ is the unit point measure at $x$, so that $\hat{\delta}_{x}(\gamma)=\langle\gamma, x\rangle$ for $\gamma \in \Gamma$.

If $S$ is a subset of the domain of a function $\varphi, \varphi \mid S$ denotes the restriction of $\varphi$ to $S$ and $\|\varphi\|_{S}=\sup \{|\varphi(s)|: s \in S\}$. On the other hand, if $V$ is a normed linear space, $\|\varphi\|_{V}$ denotes the norm of $\varphi$, regarded as an element of $V$. (These two conventions will not clash.)

2. A condition equivalent to balayage. To develop such a condition we need first to recall some facts about $B(E)$, the Banach algebra of restrictions to $E$ of Fourier transforms of measures. $B(E)=\{\varphi: \varphi=\hat{\mu} \mid E$ for some $\mu$ $\in M(G)$ \}. Because $E$ is compact, $B(E)=A(E)$, the algebra of restrictions to $E$ of Fourier transforms of integrable functions, with equality of norms so that, for $\varphi \in B(E)$,

$$
\begin{aligned}
\|\varphi\|_{B(E)} & =\inf \{\|\mu\|: \mu \in M(G) \text { and } \hat{\mu} \mid E=\varphi\} \\
& =\inf \left\{\|f\|_{1}: f \in L^{1}(G) \text { and } \hat{f} \mid E=\varphi\right\} .
\end{aligned}
$$

(See [8, p. 121].)

Let $g(E)=\left\{f: f \in L^{1}(G)\right.$ and $\left.\hat{f} \mid E=0\right\}$. Then we may view $B(E)$ as isometrically isomorphic to the quotient algebra $L^{1}(G) / G(E)$ (using for "transport des structures").

Define a linear operator $L: M(\Lambda) \rightarrow B(E)$ by $L \mu=\hat{\mu} \mid E$. By the definition of the $B(E)$-norm, $\|L\| \leqslant 1$. (Actually, $\|L\|=1$ because $\left\|L \delta_{\lambda}\right\|_{B(E)}=1$ for $\lambda \in \Lambda$.)

We pause now to make a general definition. Suppose that $X$ and $Y$ are Banach spaces and that $T: X \rightarrow Y$ is a surjective bounded linear operator. We set $K(T)=$ the infimum of constants $K$ such that for all $y \in Y$ there is some $x \in X$ with $T x=y$ and $\|x\| \leqslant K\|y\|$. By the open mapping theorem, $K(T)$ $<\infty$. If $T$ is not surjective, we set $K(T)=\infty$ and $1 / K(T)=0$.

In our situation, following Beurling, we set $K(\Lambda, E)=K(L)$. Thus balayage is possible for $(\Lambda, E)$ if and only if $K(\Lambda, E)<\infty$. (Always, $K(\Lambda, E) \geqslant 1$.)

Our major step in reformulating the question of balayage is to compute the adjoint of $L$. First, we must identify certain dualities.

Since $\Lambda$ is discrete, we may think of $M(\Lambda)$ as $l^{1}(\Lambda)$ (the space of absolutely summable functions on $\Lambda$ ). Then $M(\Lambda)^{*} \approx l^{\infty}(\Lambda)$ (the bounded complex functions on $\Lambda$ ) under the pairing $\langle\mu, \varphi\rangle=\int \varphi d \mu=\sum \varphi(\lambda) \mu(\{\lambda\})$. Since $B(E) \approx L^{1}(G) / G(E)$ we have $B(E)^{*} \approx G(E)^{\perp} \subset L^{1}(G)^{*} \approx L^{\infty}(G)$ where $g(E)^{\perp}$ is the annihilator of $g(E)$. More specifically, $g \in L^{\infty}(G)$ belongs to $g(E)^{\perp}$ if and only if $\langle f, g\rangle=\int_{G} f(x) g(x) d x=0$ for all $f \in g(E)$. This is equivalent to requiring that $\left(g^{*}\right) * f=0$ for all $f \in \mathscr{G}(E)$ where $g^{*}(x)$ $=g(-x)$. Thus, the pairing between $g \in g(E)^{\perp}$ and $\varphi \in B(E)$ is given by 


$$
\langle\varphi, g\rangle=\int_{G} g(x) f(x) d x \text { for any } f \in L^{1}(G) \text { with } \hat{f} \mid E=\varphi .
$$

This duality is more or less well known. Now, we would like to extend (2) to read

$$
\langle\varphi, g\rangle=\int_{G} g d \mu \quad \text { where } \mu \in M(G) \text { and } \hat{\mu} \mid E=\varphi .
$$

This raises the problem that various versions of $g$ which are equal locally a.e. may give different values to $\int_{G} g d \mu$. The correct generalization of (2) is

THEOREM 2.1. Suppose $\varphi \in B(E), \mu \in M(G)$ and $\hat{\mu} \mid E=\varphi$. If $g \in g(E)^{\perp}$, then there is a unique continuous (indeed, uniformly continuous) function $g_{0}$ in the $L^{\infty}$-equivalence class of $g$ and, for this function $g_{0}$,

$$
\langle\varphi, g\rangle=\int_{G} g_{0}(x) d \mu(x) .
$$

Statements similar to (but apparently not identical to) Theorem 2.1 have appeared previously, for example [8, p. 120 , especially Corollary 2].

We defer the proof while we present three lemmas.

LEMMA 2.1. Suppose $h_{0}$ is a bounded Borel function on $G, f_{0}$ is (Haar)integrable and $\mu \in M(G)$. Then

$$
h_{0} *\left(\mu * f_{0}\right)=\left(h_{0} * \mu\right) * f_{0} \quad \text { (everywhere). }
$$

Proof. [4, pp. 726-728] shows that $h_{0} * \mu$ is a Borel function on $G$ so that the right-hand side of (3) is defined. The equation itself follows readily from Fubini's theorem and the translation invariance of Haar measure.

ReMARK. The right-hand side of (3) is a bounded Borel function convolved with an integrable function and, therefore,depends only on the $L^{1}$-equivalence class of $f_{0}$. Likewise, the left-hand side depends only on the $L^{\infty}$-equivalence class of $h_{0}$. Thus, if $h \in L^{\infty}, f \in L^{1}$ and $\mu \in M$, we may write $h *(\mu * f)$ $=(h * \mu) * f$.

LEMMA 2.2. Suppose $\mu \in M(G)$ satisfies $\hat{\mu} \mid E=0$. If $g \in g(E)^{\perp}$ and $g_{0}$ is a bounded Borel function in the $L^{\infty}$-equivalence class of $g$, then $\left(g_{0}^{*}\right) * \mu=0$ locally a.e.

Proof. Suppose $f \in L^{1}(G)$. Then $\mu * f \in g(E)$. Thus, $\left(g_{0}^{*}\right) *(\mu * f)=0$. From Lemma 2.1, $\left(\left(g^{*}\right) * \mu\right) * f=0$ so that $\left(g_{0}^{*}\right) * \mu$ is a bounded Borel function which annihilates every $L^{1}$-function and so must vanish locally a.e.

LEMma 2.3. If $g \in \mathscr{G}(E)^{\perp}$, then there is a (necessarily unique) uniformly continuous function $g_{0}$ in the $L^{\infty}$-equivalence class of $g$.

Proof. This is known: $g(E)^{\perp} \subset L^{\infty}(G, E)$, those functions in $L^{\infty}(G)$ whose weak-* spectrum lies in $E$. Since $E$ is compact, the result follows; see for example, [9, pp. 140-141]. 
Now we can prove Theorem 2.1. Under the hypotheses of that theorem, there is some $f \in L^{1}(G)$ with $\hat{f} \mid E=\varphi$. Then $(\mu-f)^{\hat{}} \mid E=0$, so, letting $g_{0}$ be the uniformly continuous version of $g$ whose existence is guaranteed by Lemma 2.3 we have, by Lemma 2.2 ,

$$
\left(\left(g_{0}^{*}\right) *(\mu-f)\right)(x)=0 \text { locally a.e. in } x .
$$

Now, $\left(g_{0}^{*}\right) *(\mu-f)$ is uniformly continuous (because $\left.g_{0}^{*}\right)$ is so (4) holds for all $x$. Setting $x=0$ gives $\left(\left(g_{0}^{*}\right) * \mu\right)(0)=\left(\left(g_{0}^{*}\right) * f\right)(0)$. Thus

$$
\begin{aligned}
\langle\varphi, g\rangle & =\int_{G} f g_{0} d x=\left(\left(g_{0}^{*}\right) * f\right)(0) \\
& =\left(\left(g_{0}^{*}\right) * \mu\right)(0)=\int_{G} g_{0} d \mu
\end{aligned}
$$

proving the theorem.

Henceforth, we shall view $g(E)^{\perp}$ as a space of continuous functions on $G$, a view justified by Lemma 2.3 .

THEOREM 2.2. With $L$ as defined above, $L^{*}: g(E)^{\perp} \rightarrow l^{\infty}(\Lambda)$ is given by $L^{*} g=g \mid \Lambda$.

Proof. Suppose $\mu \in M(\Lambda) \approx l^{1}(\Lambda)$. Then, $\left\langle\mu, L^{*} g\right\rangle=\langle L \mu, g\rangle=\langle\hat{\mu} \mid E, g\rangle$ $=\int_{G} g d \mu=\sum g(\lambda) \mu(\{\lambda\})=\langle\mu, g \mid \Lambda\rangle$. Since this holds for all $\mu \in M(\Lambda)$, we have $L^{*} g=g \mid \Lambda$.

We will need a functional-analytic result (used, for example, in the study of Helson sets). We rephrase Theorem E9 (of Appendix E) of [4] for our purposes.

Theorem 2.3. Suppose $L: X \rightarrow Y$ (where $X$ and $Y$ are Banach spaces) is a bounded linear operator with adjoint $L^{*}: Y^{*} \rightarrow X^{*}$. Suppose $C>0$. Then the two following conditions are equivalent:

1. $L$ is surjective and $C=K(L)$.

2. $C\left\|L^{*} y^{*}\right\| \geqslant\left\|y^{*}\right\|$ for all $y^{*} \in Y^{*}$.

We apply this theorem to our $L: M(\Lambda) \rightarrow B(E)$. Define

$$
k^{\prime}(\Lambda, E)=\sup \left\{\|g\|_{G} /\|g\|_{\Lambda}: g \in g(E)^{\perp} \text { and } g \neq 0\right\} .
$$

(This may be $\infty$.) Applying Theorem 2.3 to $L$ gives immediately

Theorem 2.4. $K(\Lambda, E)=k^{\prime}(\Lambda, E)$.

In particular, in order for balayage to be possible for $(\Lambda, E)$, each nonzero $g \in g(E)^{\perp}$ must satisfy $g \mid \Lambda \neq 0$. (Then $\Lambda$ is said to be a set of sampling for $g(E)^{\perp}$.)

REMARK. Beurling used the open mapping theorem to show the finiteness of $K(\Lambda, E)$ when balayage is possible. In place of $g(E)^{\perp}$, however, he considered $C(G, E)$, the space of bounded continuous functions with spectra in $E$. He defined $k(\Lambda, E)$ in the same manner as our $k^{\prime}$ but using a sup over $C(G, E)$. 
instead of $9(E)^{\perp}$, and showed that if $E$ satisfies his conditions $(\alpha)$ and $(\beta)$ then $K(\Lambda, E)=k(\Lambda, E)$. Condition $(\alpha)$ is that every neighborhood, in $E$, of every point of $E$ support a measure whose Fourier transform vanishes at infinity. Condition $(\beta)$ is that $E$ be a set of spectral synthesis, i.e., that $C(G, E)$ $=g(E)^{\perp}$. Thus, Theorem 2.4 contains Beurling's result. Note that, in general, $g(E)^{\perp} \subset C(G, E)$ so that $k^{\prime}(\Lambda, E) \leqslant k(\Lambda, E)$.

3. The case of compact, open $E$. We now give an application of Theorem 2.4 to the situation where $E$ is a compact and open subset of $\Gamma$. We note that in the important case of totally disconnected groups, every compact subset can be approximated (from outside) by compact, open sets. We also remark that, for the groups of $p$-adic numbers, balayage and related matters where (in our notation) $E$ is discrete and $\Lambda$ is compact have been studied by various French workers. (See [8, p. 162ff.] and the references given in that book.)

Since $E$ is compact and open, we may write $E=\cup_{1}^{n}\left(\gamma_{k}+H\right)$ where $H$ is a compact and open subgroup of $\Gamma$ and the various cosets $\gamma_{k}+H, k=1,2$, $\ldots, n$,are distinct. (Apply, for example, [4, Theorem 7.5].)

We write $H^{\perp}$ for the annihilator of $H$, that is, $H^{\perp}=\{x \in G:\langle\gamma, x\rangle=1$ for all $\gamma \in H\} . H^{\perp}$ is a compact, open subgroup of $G$. We assume that the Haar measures $m_{G}$ and $m_{\Gamma}$ on $G$ and $\Gamma$ respectively are so normalized that the Fourier inversion theorem holds. In particular, we have $m_{\Gamma}(H) m_{G}\left(H^{\perp}\right)=1$.

THEOREM 3.1. With notation as above, if balayage is possible for $(\Lambda, E)$ then $\Lambda$ contains at least $n$ elements from every coset of $H^{\perp}$.

Proof. We prove the contrapositive. Suppose that there is some $x$ such that $\Lambda \cap\left(x+H^{\perp}\right)=\left\{\lambda_{1}, \lambda_{2}, \ldots, \lambda_{m}\right\}$ and $m<n$. Then there is a nontrivial solution $\left(d_{1}, d_{2}, \ldots, d_{n}\right)$ to the system of $m$ equations in $n$ unknowns:

$$
\sum_{k=1}^{n} d_{k}\left\langle\gamma_{k}, \lambda_{j}\right\rangle=0 ; \quad j=1,2, \ldots, m .
$$

Fixing one such solution, we define $g$ by

$$
g(t)= \begin{cases}\sum_{1}^{n} d_{k}\left\langle\gamma_{k}, t\right\rangle & \text { if } t \in x+H^{\perp}, \\ 0 & \text { if } t \notin x+H^{\perp} .\end{cases}
$$

$\int_{G}|g|^{2}=m_{G}\left(H^{\perp}\right) \sum\left|d_{k}\right|^{2} \neq 0$ so $g$ does not vanish identically. On the other hand, $g\left(\lambda_{j}\right)=0$ for $j=1,2, \ldots, m$ so that $g \mid \Lambda=0$. If we can show that $g \in \mathscr{G}(E)^{\perp}$, we will thus be able to conclude that $k^{\prime}(\Lambda, E)=\infty$, proving the theorem. First we note that $g$ is continuous since $x+H^{\perp}$ is open. Next, we look at $\check{g}(\gamma)=\hat{g}(-\gamma)$.

$$
\begin{aligned}
\check{g}(\gamma) & =\sum d_{k} \int_{x+H^{\perp}}\left\langle\gamma_{k}-\gamma, t\right\rangle d t \\
& =\Sigma d_{k}\left\langle\gamma_{k}-\gamma, x\right\rangle \int_{H^{\perp}}\left\langle\gamma_{k}-\gamma, t\right\rangle d t .
\end{aligned}
$$


Now, $\int_{H^{\perp}}\left\langle\gamma_{k}-\gamma, t\right\rangle d t$ can be nonzero only if $\gamma_{k}-\gamma$ is the trivial character on $H^{\perp}$, that is, only if $\gamma \in \gamma_{k}+H$. Thus, $\check{g}$ vanishes off $E$. In particular, $g=\left(g^{*}\right)^{n}$ which justifies our concluding that if $f \in g(E)$, then $\int_{G} f g$ $=\int_{G} f\left(g^{\circ}\right)^{\wedge}=\int_{\Gamma} \hat{f} \check{g}=0$ because $\hat{f}$ vanishes on $E$. Thus, $g \in G(E)^{\perp}$, proving the theorem.

REMARK. Suppose we define

$$
\Delta_{-}(\Lambda)=\lim \inf \frac{\#\left(\Lambda \cap K_{j}\right)}{m_{G}\left(K_{j}\right)}
$$

where each $K_{j}$ is the union of (finitely many) cosets of $H^{\perp}$, the $K_{j}$ 's increase to $G$ and $\#(S)$ is the number of elements in $S . \Delta_{-}$is a not very intrinsic density analogous to $D_{-}$. If $K_{j}$ is the union of $m_{j}$ cosets of $H^{\perp}$, then $m_{G}\left(K_{j}\right)=m_{j} m_{G}\left(H^{\perp}\right)$ and, for balayage to be possible, we must have $\#\left(\Lambda \cap K_{j}\right) \geqslant m_{j} n$ for all $j$. In this case, $\Delta_{-}(\Lambda) \geqslant m_{j} n / m_{j} m_{G}\left(H^{\perp}\right)$ $=n m_{\Gamma}(H)=m_{\Gamma}(E)$, a relation formally analogous to (1). (Here, unlike (1), we cannot require strict inequality, see Theorem 3.2.)

Because of algebraic complications, the converse to Theorem 3.1 is false unless $n=1$, as the following example shows. Let $\Gamma(\approx G)$ be a finite group with a proper, nontrivial subgroup $K$. Let $\gamma_{0} \in K$ be nonzero, let $H=\{0\}$ and let $E=\left\{0, \gamma_{0}\right\}=H \cup\left(\gamma_{0}+H\right)$, so that $n=2$. Let $x_{0}$ be a nonzero element of $K^{\perp}$ and let $\Lambda=\left\{0, x_{0}\right\} . \Lambda$ meets each coset (there's only one) of $H^{\perp}=G$ twice. Let $f(t)=1-\left\langle-\gamma_{0}, t\right\rangle$. A simple computation shows that $\hat{f}(\gamma)=0$ unless $\gamma \in E$ so one verifies readily that $f \in \mathscr{g}(E)^{\perp}$. However, $f$ is nontrivial and $f \mid \Lambda=0$ because $\left\langle-\gamma_{0}, x_{0}\right\rangle=1$. Thus $k^{\prime}(\Lambda, E)=\infty$. Such examples can be lifted to infinite LCA groups having two proper, compact and open subgroups.

We do have, however

THEOREM 3.2. If $H$ is a compact, open subgroup of $\Gamma$ then balayage is possible for $(\Lambda, H)$ if and only if $\Lambda$ meets every coset of $H^{\perp}$.

Proof. The "only if" follows from Theorem 3.1. Let

$$
h(t)= \begin{cases}m_{\Gamma}(H) & \text { if } t \in H^{\perp}, \\ 0 & \text { if } t \notin H^{\perp} .\end{cases}
$$

One verifies easily that $\hat{h}$ is the characteristic function of $H$. If $g \in g(H)^{\perp}$, Lemma 2.2 insures that $\left(g^{*}\right) *\left(\delta_{0}-h\right)=0$ so that

$$
g(x)=\int_{H^{\perp}} g(x-t) m_{\Gamma}(H) d t .
$$

Thus $g$ is constant on the cosets of $H^{\perp}$. If $\Lambda$ meets each coset of $H^{\perp}$ then $\|g\|_{G}=\|g\|_{\Lambda}$. Thus, $k^{\prime}(\Lambda, H)=1$, proving the theorem.

REMARK. A more general result of this kind, where $H$ need not be compact, can be proved using a measurable choice argument [10, Theorem 5.2]. 
4. General perturbation results. For the purpose of comparing different discrete sets, we change our viewpoint slightly from that of $\$ 2$. Let $\Lambda$ $=\left\{\lambda_{i}\right\}_{i \in I}$ where the indexing puts $\Lambda$ into one-to-one correspondence with the index set $I$. Since $\Lambda$ is discrete, there is an isometry between $l^{1}(I)$ and $M(\Lambda)$ which makes the function $\alpha \in l^{1}(I)$ correspond to the measure $\sum \alpha(i) \delta_{\lambda_{1}}$.

Define $T_{\Lambda}: l^{1}(I) \rightarrow B(E)$ by $T_{\Lambda} \alpha=\left(\Sigma \alpha(i) \delta_{\lambda_{i}}\right)^{\wedge} \mid E$. More explicitly,

$$
\left(T_{\Lambda} \alpha\right)(\gamma)=\sum \alpha(i)\left\langle\gamma, \lambda_{i}\right\rangle \text { for } \gamma \in E .
$$

$T_{\Lambda}$ is a bounded linear operator of norm 1 and balayage is possible for $(\Lambda, E)$ if and only if $T_{\Lambda}$ is surjective. Indeed, we easily see that $K(\Lambda, E)=K\left(T_{\Lambda}\right)$, in the notation of $\$ 2$.

We shall use the following well-known result, one of whose proofs follows from the obvious iterative scheme. (See [1, Theorem 1.2] or [10, Proposition 4.1].) The idea of this result has also been used by Beurling to solve a related interpolation problem.

THEOREM 4.1. Suppose $X$ and $Y$ are Banach spaces and $S$ and $T$ are bounded linear operators from $X$ to $Y$. Then,

$$
|1 / K(S)-1 / K(T)| \leqslant\|S-T\| .
$$

In particular, if $T$ is surjective and $\|S-T\|<1 / K(T)$, then $S$ is surjective.

Suppose now that $\Lambda^{\prime}$ is another closed, discrete set in $G$ and for each $i \in I$ we have an element $\lambda_{i}^{\prime} \in \Lambda^{\prime}$. Here (unlike the case for $\Lambda$ ) we do not assume that the $\lambda_{i}^{\prime}$ are distinct or that $\left\{\lambda_{i}^{\prime}\right\}_{i \in I}$ is all of $\Lambda^{\prime}$.

Define $S_{\Lambda^{\prime}}: l^{l}(I) \rightarrow B(E)$ by $S_{\Lambda^{\prime}} \alpha=\left(\sum \alpha(i) \delta_{\lambda_{i}^{\prime}}\right) \mid E$. As above, if $S_{\Lambda^{\prime}}$ is surjective, then balayage is possible for $\left(\Lambda^{\prime}, E\right)$. Because of the lack of assumptions on the indexing of the $\lambda_{i}^{\prime}$ we have only $K\left(\Lambda^{\prime}, E\right) \leqslant K\left(S_{\Lambda^{\prime}}\right)$.

To apply Theorem 4.1, we need an estimate of $\left\|T_{\Lambda}-S_{\Lambda^{\prime}}\right\|$. If $x \in G$, we write $\langle\cdot, x\rangle$ for the function $\gamma \mapsto\langle\gamma, x\rangle$. (If we denoted this function by $x, x+y$ would have a double meaning.)

LEMMA 4.1 .

$$
\left\|T_{\Lambda}-S_{\Lambda^{\prime}}\right\| \leqslant \sup \left\{\left\|1-\left\langle\cdot, \lambda_{i}^{\prime}-\lambda_{i}\right\rangle\right\|_{B(E)}: i \in I\right\} .
$$

Proof. Given $\alpha \in l^{1}(I)$ and $\gamma \in E$ we have

$$
\begin{aligned}
\left(T_{\Lambda} \alpha-S_{\Lambda^{\prime}} \alpha\right)(\gamma) & =\sum \alpha(i)\left(\left\langle\gamma, \lambda_{i}\right\rangle-\left\langle\gamma, \lambda_{i}^{\prime}\right\rangle\right) \\
& =\sum \alpha(i)\left\langle\gamma, \lambda_{i}\right\rangle\left(1-\left\langle\gamma, \lambda_{i}^{\prime}-\lambda_{i}\right\rangle\right) .
\end{aligned}
$$

Now $\left\|\left\langle\cdot, \lambda_{i}\right\rangle\right\|_{B(E)}=1$ and $B(E)$ is a normed algebra, so

$$
\begin{aligned}
\left\|T_{\Lambda} \alpha-S_{\Lambda^{\prime}} \alpha\right\|_{B(E)} & \leqslant \sum|\alpha(i)|\left\|1-\left\langle\cdot, \lambda_{i}^{\prime}-\lambda_{i}\right\rangle\right\|_{B(E)} \\
& \leqslant\|\alpha\|_{l^{1}(I)} \sup \left\{\left\|1-\left\langle\cdot, \lambda_{i}^{\prime}-\lambda_{i}\right\rangle\right\|_{B(E)}: i \in I\right\},
\end{aligned}
$$

from which the lemma follows. 
LEMMA 4.2. Given a compact set $E \subset \Gamma$, and $\varepsilon>0$, there is a neighborhood $U$ of 0 in $G$ such that

$$
\sup \left\{\|1-\langle\cdot, x\rangle\|_{B(E)}: x \in U\right\}<\varepsilon .
$$

Proof. Since $E$ is compact, there is some $f \in L^{1}(G)$ with $\hat{f} \mid E=1$. We recall that $\lim _{x \rightarrow 0}\left\|f-f_{x}\right\|_{1}=0$, where $f_{x}(t)=f(t+x)$. There is, thus, a neighborhood $U$ of 0 such that $\sup \left\{\left\|f-f_{x}\right\|_{1}: x \in U\right\}<\varepsilon$. Now $\hat{f}_{x}(\gamma)$ $=\langle\gamma, x\rangle \hat{f}(\gamma)=\langle\gamma, x\rangle$ for $\gamma \in E$. Thus, $\left(f-f_{x}\right)^{\hat{\lambda}}=1-\langle\cdot, x\rangle$ on $E$ so that $\|1-\langle\cdot, x\rangle\|_{B(E)} \leqslant\left\|f-f_{x}\right\|_{1}$ and the lemma follows.

THEOREM 4.2. Suppose balayage is possible for $(\Lambda, E)$. Then there is a neighborhood $U$ of 0 in $G$ with the following property: if $\Lambda^{\prime}$ is any discrete set such that for every $\lambda \in \Lambda$ there is some $\lambda^{\prime} \in \Lambda^{\prime}$ with $\lambda^{\prime}-\lambda \in U$, then balayage is possible for $\left(\Lambda^{\prime}, E\right)$.

Proof. Let $U$ be as in Lemma 4.2 with $\varepsilon=1 / K(\Lambda, E)$. Given $\Lambda^{\prime}$ as in the statement of the theorem, it is clear that $\Lambda$ and $\Lambda^{\prime}$ may be indexed as in the discussion above. Then, by Lemma 4.1, $\left\|T_{\Lambda}-S_{\Lambda^{\prime}}\right\|<1 / K(\Lambda, E)=1 / K\left(T_{\Lambda}\right)$ so, by Theorem 4.1, $S_{\Lambda^{\prime}}$ is surjective and balayage is possible for $\left(\Lambda^{\prime}, E\right)$.

One would like to have $U$ as large as possible. To this end, we need a good estimate of $\|1-\langle\cdot, x\rangle\|_{B(E)}$. We take up this problem for Euclidean spaces next.

5. The Euclidean case. We deal first with $G=R$, the real line, so that $\Gamma \approx R$ also under the pairing $\langle\gamma, x\rangle=\exp (i \gamma x)$.

We note that if $E \subset F$ and $\varphi \in B(F)$ then $\varphi \mid E \in B(E)$ and $\|\varphi \mid E\|_{B(E)}$ $<\|\varphi\|_{B(F)}$. We shall exploit this remark by considering intervals containing $E$.

We quote a result which we shall need again in $\$ 6$. This appears in a different form and context on pp. 107 and 108 of [8].

Lemma 5.1. Suppose $b>0$ and $|x| b<\pi$. Define

$$
z_{k}=\frac{x}{2}-\left(\frac{2 k+1}{2 b}\right) \pi \text { for } k=0, \pm 1, \pm 2, \ldots
$$

Then there is a measure $\mu=\Sigma c_{k} \delta_{z_{k}}$ such that

$$
\begin{aligned}
\|\mu\| & =\Sigma\left|c_{k}\right|=2 \sin (|x| b / 2) \quad \text { and } \\
\hat{\mu}(\gamma) & =1-\exp (i \gamma x) \quad \text { for }|\gamma| \leqslant b .
\end{aligned}
$$

To make our formulas simpler, we define

$$
s(t)= \begin{cases}2 \sin (|t| / 2) & \text { if }|t| \leqslant \pi \\ 2 & \text { if }|t| \geqslant \pi\end{cases}
$$


LEMMA 5.2. $\|1-\langle\cdot, x\rangle\|_{B([-b, b])}=s(x b)$.

Proof. $s(x b)=\|1-\langle\cdot, x\rangle\|_{[-b, b]} \leqslant\|1-\langle\cdot, x\rangle\|_{B([-b, b])}$. The result of Lemma 5.1 and the observation that $\|1-\langle\cdot, x\rangle\|_{B} \leqslant\|1\|_{B}+\|\langle\cdot, x\rangle\|_{B}=2$ complete the proof.

THEOREM 5.1. Suppose balayage is possible for $(\Lambda, E)$. Let $2 b=\operatorname{diameter}(E)$ and suppose $a>0$ is such that $s(a b)<1 / K(\Lambda, E)$.

Then, if $\Lambda^{\prime}$ is a discrete set such that for every $\lambda \in \Lambda$ there is some $\lambda^{\prime} \in \Lambda^{\prime}$ with $\left|\lambda^{\prime}-\lambda\right| \leqslant a$, it follows that balayage is possible for $\left(\Lambda^{\prime}, E\right)$ and that

$$
1 / K(\Lambda, E)-1 / K\left(\Lambda^{\prime}, E\right) \leqslant s(a b)
$$

Proof. Balayage is possible for $(\Lambda, E)$ if and only if balayage is possible for $(\Lambda, E+\gamma)$. Thus, we may assume that $E \subset[-b, b]$. Let $U=[-a, a]$. Lemma 3.2 gives

$$
\begin{aligned}
\sup \left\{\|1-\langle\cdot, x\rangle\|_{B(E)}: x \in U\right\} \\
\quad \leqslant \sup \left\{\|1-\langle\cdot, x\rangle\|_{B([-b, b])}: x \in U\right\} \\
\quad=s(a b)<1 / K(\Lambda, E) .
\end{aligned}
$$

Arguing as in Theorem 4.2, we obtain the result. The inequality follows from Theorem 4.1 and the fact that $K\left(\Lambda^{\prime}, E\right) \leqslant K\left(S_{\Lambda^{\prime}}\right)$.

REMARK. Beurling proved a similar result with $s(a b)$ replaced by $a b>s(a b)$. He worked with $C(R, E)$ and assumed his conditions $(\alpha)$ and $(\beta)$. Actually, his proof, which makes use of Bernstein's inequality, carries over verbatim to $g(E)^{\perp}$ without these conditions. We need the present technique, however, to deal with more general groups and, in \$6, with a different type of perturbation. Our proof of Lemma 6.1 is an adaptation and illustration of Beurling's argument.

We now consider the case where $G=R^{n}$ so $\Gamma=R^{n}$ also. We adopt the notation $\mathrm{x}=\left(x_{1}, x_{2}, \ldots, x_{n}\right)$ and $\gamma=\left(\gamma_{1}, \gamma_{2}, \ldots, \gamma_{n}\right)$ for elements of $G$ and $\Gamma$ respectively so that $\langle\gamma, \mathbf{x}\rangle=\exp \left(i \sum \gamma_{k} x_{k}\right)=\exp (i \gamma \cdot \mathbf{x})$. If $\mathbf{b}=\left(b_{1}\right.$, $\left.b_{2}, \ldots, b_{n}\right)$ has nonnegative entries then we set

$$
I(\mathbf{b})=\left\{\gamma:\left|\gamma_{k}\right| \leqslant b_{k} \text { for } k=1,2, \ldots, n\right\} .
$$

LeMma 5.3. $\|1-\langle\cdot, x\rangle\|_{B(I(b))} \leqslant \sum_{1}^{n} s\left(x_{k} b_{k}\right)$.

Proof. For $k=1,2, \ldots, n$ we define a measure $\mu_{k}$ as follows:

If $\left|x_{k}\right| b_{k}<\pi$ we let $\mu$ be the measure on $R$ constructed as in Lemma 5.1 with $b$ replaced by $b_{k}$ and $x$ by $x_{k}$.

If $\left|x_{k}\right| b_{k} \geqslant \pi$ we let $\mu=\delta_{0}-\delta_{x_{k}}$.

$\mu_{k}$ is then defined by the requirement that 


$$
\int_{R^{n}} f(\mathbf{v}) d \mu_{k}(\mathbf{v})=\int_{R} f\left(0, \ldots, 0, v_{k}, 0, \ldots, 0\right) d \mu\left(v_{k}\right)
$$

for reasonable (say bounded and continuous) functions $f$. Thus, $\hat{\mu}_{k}(\gamma)=1$ $-\exp \left(i \gamma_{k} x_{k}\right)$ if $\left|\gamma_{k}\right| \leqslant b_{k}$ and $\left\|\mu_{k}\right\|=\|\mu\|=s\left(x_{k} b_{k}\right)$. Thus, in the special case where $\mathbf{x}=\left(0, \ldots, 0, x_{k}, 0, \ldots, 0\right)$, we have shown that $\|1-\langle\cdot, \mathbf{x}\rangle\|_{B(I(b))}$ $\leqslant s\left(x_{k} b_{k}\right)$.

Now, let $\mathbf{x}=\left(x_{1}, x_{2}, \ldots, x_{n}\right)$ and set $\mathbf{v}_{1}=0$ and $\mathbf{v}_{k}=\left(x_{1}, x_{2}, \ldots, x_{k-1}\right.$, $0, \ldots, 0)$ for $k=2,3, \ldots, n$ and set $w_{k}=\left(0, \ldots, 0, x_{k}, 0, \ldots, 0\right)$. Then

$$
1-\langle\gamma, \mathbf{x}\rangle=\sum_{1}^{n}\left\langle\gamma, \mathbf{v}_{k}\right\rangle\left(1-\left\langle\gamma, \mathbf{w}_{k}\right\rangle\right)
$$

$\left\|\left\langle\cdot, v_{k}\right\rangle\right\|_{B}=1$ and the $w_{k}$ are of the special form for which we already have our estimate. Thus

$$
\|1-\langle\cdot, \mathbf{x}\rangle\|_{B(I(b))} \leqslant \sum\left\|1-\left\langle\cdot, \mathbf{w}_{k}\right\rangle\right\|_{B(I(b))} \leqslant \sum s\left(x_{k} b_{k}\right) .
$$

REMARK. In the case where $\Gamma=\Gamma_{1} \times \Gamma_{2} \times \cdots \times \Gamma_{n}$, the same idea would allow us to estimate $\|1-\langle\cdot, \mathbf{x}\rangle\|_{B(E)}$ where $E=E_{1} \times E_{2} \times \cdots \times E_{n}$, if we had estimates for each $\left\|1-\left\langle\cdot, x_{k}\right\rangle\right\|_{B\left(E_{k}\right)}$.

THEOREM 5.2. Suppose balayage is possible for $(\Lambda, E)$ where $E$ (or some translate of $E) \subset I(b)$. Suppose a has positive entries and

$$
\sum s\left(a_{k} b_{k}\right)<1 / K(\Lambda, E)
$$

(which is certainly the case if $\mathbf{a} \cdot \mathbf{b}<1 / K(\Lambda, E)$ ).

If $\Lambda^{\prime}$ is a discrete set such that for every $\lambda \in \Lambda$ there is some $\lambda^{\prime} \in \Lambda^{\prime}$ with $\lambda^{\prime}-\lambda \in I(\mathrm{a})$, then balayage is possible for $\left(\Lambda^{\prime}, E\right)$ and

$$
\frac{1}{K(\Lambda, E)}-\frac{1}{K\left(\Lambda^{\prime}, E\right)} \leqslant \sum s\left(a_{k} b_{k}\right)
$$

Proof. This follows from Lemma 5.3 exactly as Theorem 5.1 follows from Lemma 5.2.

6. Balayage with sparse sets of frequencies. Beurling's work on balayage dealt with the case where $\Lambda$ has positive density. In this section, we go to the opposite extreme. We confine ourselves to the case $G=R$.

Definition 6.1. $E$ is called a BAS set (for balayage with arbitrarily sparse frequencies) if the following condition holds:

Given any sequence $h_{1}, h_{2}, \ldots$ of real-valued functions, $h_{n}$ having $n-1$ real arguments (so that $h_{1}$ is a constant) there is a closed, discrete set $\Lambda=\left\{\lambda_{n}\right\}_{1}^{\infty}$ with $\lambda_{n} \geqslant h_{n}\left(\lambda_{1}, \lambda_{2}, \ldots, \lambda_{n-1}\right)$ for all $n$ and with balayage possible for $(\Lambda, E)$.

We adapt our previous techniques to prove 
THEOREM 6.1. Suppose $E$ is compact and there is a number $\delta<1$ such that for every $M$ there is a $t \geqslant M$ with $\|1-\langle\cdot, t\rangle\|_{B(E)} \leqslant \delta$. Then $E$ is a BAS set. (We could write our condition as $\lim _{\inf _{t \rightarrow \infty}}\|1-\langle\cdot, t\rangle\|_{B(E)}<1$.)

Before we prove this theorem, we need to know that if $E$ is a compact set then there is a discrete set $S$ with $K(S, E)$ arbitrarily close to 1 . We give a proof of this fact using the method (referred to after Theorem 5.1) which Beurling used to study perturbation.

LEMMA 6.1. Let $Z$ denote the integers. If $a>0, b>0$, and $a b<2$, then

$$
K(a Z,[-b, b]) \leqslant\left(1-\frac{1}{2} a b\right)^{-1} \text {. }
$$

Proof. We prove the result for $k^{\prime}$. Suppose that $\varphi \in g([-b, b])^{\perp}$. Then the spectrum of $\varphi$ lies in $[-b, b]$ so, by Bernstein's inequality (for derivatives of entire functions of exponential type)

$$
\left|\varphi(x)-\varphi\left(x^{\prime}\right)\right| \leqslant b\left|x-x^{\prime}\right|\|\varphi\|_{R}
$$

for any $x, x^{\prime} \in R$. (See, for example, [8, p. 149].)

Now, given $x \in R$ there is some $\lambda \in a Z$ with $|x-\lambda| \leqslant a / 2$. Thus, $|\varphi(x)-\varphi(\lambda)| \leqslant b(a / 2)\|\varphi\|_{R}$ whence $|\varphi(x)| \leqslant|\varphi(\lambda)|+(a b / 2)\|\varphi\|_{R}$ and it follows that $\|\varphi\|_{R}(1-a b / 2) \leqslant\|\varphi\|_{a z}$ so that

$$
k^{\prime}(a Z,[-b, b])=\sup \left\{\|\varphi\|_{R} /\|\varphi\|_{a Z}\right\} \leqslant(1-a b / 2)^{-1} .
$$

REMARK. This result is not sharp because (by (1)) $K(a Z,[-b, b])<\infty$ if $a b$ $<\pi$.

We now prove Theorem 6.1. For some $b, E \subset[-b, b]$. Pick $a$ such that $\delta<1-a b / 2$. Enumerate $a Z$ as a sequence, $a Z=\left\{x_{n}\right\}_{1}^{\infty}$. Let functions $h_{n}$ as in Definition 6.1 be given.

Proceeding inductively, by the hypothesis on $E$ there is, at each step, some $t_{n} \geqslant h_{n}\left(\lambda_{1}, \lambda_{2}, \ldots, \lambda_{n-1}\right)-x_{n}$ with $\left\|1-\left\langle\cdot, t_{n}\right\rangle\right\|_{B(E)} \leqslant \delta$. Set $\lambda_{n}=t_{n}+x_{n}$; by increasing the $t_{n}$ if necessary we may insure that the $\lambda_{n}$ are distinct and that $\Lambda=\left\{\lambda_{n}\right\}$ is discrete.

By Lemmas 4.1 and 6.1,

$$
\begin{aligned}
\left\|T_{\Lambda}-T_{a Z}\right\| & \leqslant \sup \left\{\left\|1-\left\langle\cdot, \lambda_{n}-x_{n}\right\rangle\right\|_{B(E)}\right\} \\
& =\sup \left\{\left\|1-\left\langle\cdot, t_{n}\right\rangle\right\|_{B(E)}\right\} \leqslant \delta<1-a b / 2 \\
& \leqslant 1 / K(a Z,[-b, b]) \leqslant 1 / K(a Z, E)=1 / K\left(T_{a Z}\right) .
\end{aligned}
$$

Since $T_{a Z}$ is surjective, Theorem 4.1 implies that $T_{\Lambda}$ is surjective and our theorem follows.

REMARK. We have $K(\Lambda, E) \leqslant(1-\delta-a b / 2)^{-1}$ so we can make $K(\Lambda, E)$ $\left\langle(1-\delta)^{-1}+\varepsilon\right.$ for any $\varepsilon>0$.

We recall that $E$ is called a Dirichlet set if there is a sequence $t_{n}$ 
$\rightarrow \infty$ with $\left\|1-\left\langle\cdot, t_{n}\right\rangle\right\|_{E} \rightarrow 0$. $E$ is called a Helson set if $B(E)=C(E)$, the algebra of all continuous complex functions on $E$. We shall see shortly that every Dirichlet set is a BAS set and that there are BAS sets which are neither Dirichlet nor Helson. To these ends, we need an estimate.

THEOREM 6.2. If $\|1-\langle;, x\rangle\|_{E}=\varepsilon$ then

$$
\|1-\langle\cdot, x\rangle\|_{B(E)} \leqslant 2 \sin (\pi / 2 p)
$$

where $p$ is the largest odd integer such that

$$
s^{-1}(\varepsilon)=2 \sin ^{-1}(\varepsilon / 2) \leqslant \pi / p
$$

In particular,

$$
\|1-\langle\cdot, x\rangle\|_{B(E)} \leqslant 2\|1-\langle\cdot, x\rangle\|_{E}
$$

Proof. We assume for convenience that $x>0$.

$|1-\exp (i \gamma x)| \leqslant \varepsilon$ iff $|\sin (\gamma x / 2)| \leqslant \varepsilon / 2$ which occurs iff there is an integer $k$ with $|\gamma x-2 k \pi| \leqslant 2 \sin ^{-1}(\varepsilon / 2)$. Thus, $\|1-\langle\cdot, x\rangle\|_{E} \leqslant \varepsilon$ iff

$$
E \subset \bigcup_{k=-\infty}^{\infty}\left[\frac{2 k \pi}{x}-\frac{s^{-1}(\varepsilon)}{x}, \frac{2 k \pi}{x}+\frac{s^{-1}(\varepsilon)}{x}\right] .
$$

Suppose this to be the case and let $p$ be the largest odd integer with $s^{-1}(\varepsilon) \leqslant \pi / p$. Then

$$
E \subset \bigcup_{k=-\infty}^{\infty}\left[\frac{2 k \pi}{x}-\frac{\pi}{p x}, \frac{2 k \pi}{x}+\frac{\pi}{p x}\right] .
$$

We return now to the notation of Lemma 5.1 and set $b=\pi / p x$ so that

$$
z_{k}=\frac{x}{2}-((2 k+1) / 2 b) \pi=N_{k} x
$$

where $N_{k}$ is an integer. If we now set $\mu=\sum c_{k} \delta_{z_{k}}$ as in Lemma 5.1 we see, on one hand, that

$$
\hat{\mu}(\gamma)=1-\exp (i \gamma x) \text { for }|\gamma| \leqslant \pi / p x
$$

and, on the other hand, that

$$
\hat{\mu}(\gamma)=\sum c_{k} \exp \left(i N_{k} x \gamma\right)
$$

has period $2 \pi / x$. These observations, together with (5), show that $\hat{\mu}(\gamma)$ $=1-\exp (i \gamma x)$ for all $\gamma \in E$. Since $\|\mu\|=\Sigma\left|c_{k}\right|=s(x b)=2 \sin (\pi / 2 p)$, the result follows.

The idea of using odd integers $p$ comes, partly, from [8, p. 107].

There now follows directly from the last two theorems: 
COROLlary 6.1. Every Dirichlet set is a BAS set.

The idea of Theorem 6.2 will now be used to construct a class of BAS sets. We would like to construct a set $E$ in such a manner that there is a sequence $x_{n} \rightarrow \infty$ with

$$
\left\|1-\left\langle\cdot, x_{n}\right\rangle\right\|_{E} \leqslant \delta
$$

for some small $\delta$, but we do not want $\left\|1-\left\langle\cdot, x_{n}\right\rangle\right\|_{E}$ to approach 0 because we want $E$ not to be a Dirichlet set.

Now, as we have seen, for a fixed $n(6)$ is equivalent to:

$$
E \subset \bigcup_{k=-\infty}^{\infty}\left[\frac{2 k \pi}{x_{n}}-\frac{s^{-1}(\delta)}{x_{n}}, \frac{2 k \pi}{x_{n}}+\frac{s^{-1}(\delta)}{x_{n}}\right]=F_{n},
$$

say. We would like to take for $E$ a compact subset of $\cap_{1}^{\infty} F_{n}$, but the $x_{n}$ must be picked coherently to prevent this set from being too small.

We pick $\delta<1$ so that $N=(2 \pi+r) / r$ is integral where $r=s^{-1}(\delta)$. Let $x_{0}>0$ be arbitrary and let $x_{n}=N^{n} x_{0}$ for $n=1,2, \ldots$ We set

$$
\begin{aligned}
& E_{0}=\left[-\frac{r}{x_{0}}, \frac{r}{x_{0}}\right], \\
& E_{1}=\left[-\frac{2 \pi}{x_{1}}-\frac{r}{x_{1}}, \frac{2 \pi}{x_{1}}+\frac{r}{x_{1}}\right] \cup\left[\frac{r}{x_{1}}, \frac{r}{x_{1}}\right] \cup\left[\frac{2 \pi}{x_{1}}-\frac{r}{x_{1}} \frac{2 \pi}{x_{1}}+\frac{r}{x_{1}}\right]
\end{aligned}
$$

and, in general, $E_{n}=F_{n} \cap E_{n-1}$ with $F_{n}$ as in (7).

Let $L=2 r / x_{0}$. Because of the choice of $\delta$ and the definitions of $N$ and $x_{n}$, it follows that $E_{n}$ is the union of $3^{n}$ intervals each of length $L N^{-n}$. Moreover, each interval $I$ in $E_{n-1}$ is replaced in $E_{n}$ by 3 intervals, each $1 / N$ times the length of $I$, one starting at the left endpoint of $I$, one centered at the center of $I$ and one ending at the right endpoint of $I$.

Thus, if we define $E=\cap_{0}^{\infty} E_{n}$, then $E$ is a compact set of the sort called by Kahane and Salem [6, pp. 16-17] "un parfait homogène" of type $(3,1 / N)$.

THEOREM 6.3. Let $\delta=2 \sin (\pi / 2 q)$ where $2 q \geqslant 10$ is an integer. Then $E$ as constructed above is a BAS set which is neither a Helson set nor a Dirichlet set.

Proof. Note, first, that $s^{-1}(\delta)=\pi / q$ so that $N=2 q+1$ is integral. By construction, we have $\left\|1-\left\langle\cdot, x_{n}\right\rangle\right\|_{E}=\delta$ for all $n$, and thus Theorem 6.2 implies that $\left\|1-\left\langle\cdot, x_{n}\right\rangle\right\|_{B(E)} \leqslant 2 \sin (\pi / 10)<1$. By Theorem 6.1, $E$ is, therefore, a BAS set.

It is known that no "parfait symétrique" is a Helson set $[6$, p. 146]. $E$ contains such a Cantor-like symmetric set (formed by excluding from $E$ the "middle $1 / N$ 's") and is, thus, not Helson.

That no "parfait homogène" is a Dirichlet set follows readily from the fact that for any Dirichlet set $S$ there are sequences $a_{j} \rightarrow 0$ and $b_{j} \rightarrow \infty$ such that, for each $j, S$ can be covered by intervals of length $a_{j}$ with the distances 
between successive covering intervals all less than $a_{j} b_{j}$. (See $[5$, p. 90].)

REMARKS. 1. At the cost of some simplicity in the construction of $E$, we could replace $\delta$ and $N$ by $\delta_{n}$ and $N_{n}$, varying with $n$, taking care to keep the $N_{n}$ integral and bounded.

2. The same lacunarity conditions as in the definition of BAS sets have been considered by Kahane in a different, though related, context. See [6, p. 160] where it is stated that symmetric sets similar to the set $E$ constructed above have a strong uniqueness property. Indeed, they are contained in the zero-sets of nontrivial, arbitrarily sparse, absolutely convergent trigonometric series. (A similar result follows from our methods by looking at the difference between a fixed character $\lambda_{0}$ and a series involving a sparse set of frequencies distinct from $\lambda_{0}$ and converging on $E$ to $\lambda_{0}$.)

Thinness itself does not imply the BAS property. We recall that $E$ is called an $M_{0}$-set if $E$ supports a measure whose Fourier transform vanishes at infinity; otherwise $E$ is a $U_{0}$-set. $\Lambda$ is said to be relatively dense in the group $G$ if there is a compact set $K$ with $\Lambda+K=G$. Returning to general LCA groups we have

THEOREM 6.4. If $E$ is an $M_{0}$-set and balayage is possible for $(\Lambda, E)$ then $\Lambda$ is relatively dense. In particular, every $B A S$ set is a $U_{0^{-}}$set.

Proof. It suffices, by Theorem 2.4 , to suppose that $\Lambda$ is not relatively dense and show that $k^{\prime}(\Lambda, E)=\infty$.

Let $\mu \in M(E)$ be a fixed, nonzero measure such that $\lim _{x \rightarrow \infty} \hat{\mu}(x)=0$. Suppose $\varepsilon>0$. Then, there is a compact set $K$ such that $|\hat{\mu}(x)|<\varepsilon$ if $x \notin K$.

By assumption, $-K+\Lambda \neq G$. Let $x_{0} \notin-K+\Lambda$, and define $\sigma$ by $d \sigma(\gamma)$ $=\left\langle\gamma,-x_{0}\right\rangle d \mu(\gamma)$. Then $\sigma \in M(E)$ and $\hat{\sigma}(x)=\hat{\mu}\left(x-x_{0}\right)$. Thus, if $\lambda \in \Lambda$ then $\lambda-x_{0} \notin K$ so that $|\hat{\sigma}(\lambda)|=\left|\hat{\mu}\left(\lambda-x_{0}\right)\right|<\varepsilon$. Now, $\hat{\sigma} \in g(E)^{\perp}$ because if $h \in g(E)$ then $\int_{G} h \hat{\sigma} d x=\int_{\Gamma} \hat{h} d \sigma=\int_{E} 0=0$. Also, $\|\sigma\|_{G}=\|\mu\|_{G}$.

Thus $k^{\prime}(\Lambda, E) \geqslant\|\hat{\sigma}\|_{G} /\|\hat{\sigma}\|_{\Lambda} \geqslant\|\hat{\mu}\|_{G} / \varepsilon$. Since $\varepsilon$ is arbitrary, $k^{\prime}(\Lambda, E)=\infty$, proving the theorem.

We can now see that not every "parfait homogène" $E$ is a BAS set, even if its ratio of dissection $\theta(=1 / N$ in our case $)$ is small enough. Indeed, if $1 / \theta$ is not a Pisot number (an algebraic integer all of whose conjugates have modulus less than 1) then $E$ is an $M_{0}$-set [6, Chapter VI]. It would be interesting to know whether some homogeneous $U_{0}$-set with $1 / \theta$ an irrational Pisot number is a BAS set.

COROLlaRy 6.2. If balayage is possible for $(\Lambda, E)$ where $E$ has positive Haar measure, then $\Lambda$ is relatively dense.

Proof. Such an $E$ is a $M_{0}$-set.

COROLLARY 6.3. If $G$ is a nondiscrete, metrisable LCA group, then there is a perfect, strongly independent set $E \subset G$ with the property that in order for balayage to be possible for $(\Lambda, E), \Lambda$ must be relatively dense. 
Proof. Varopoulos [11, Theorem $R$ ] has shown that $G$ contains such a set $E$ which is also an $M_{0}$-set.

ADDED IN PROOF. We have recently realized that many results of our $\$ \S 2$ and 6 were known to Kahane. Indeed [5, p. 150] gives a result equivalent to our Theorem 2.4 but phrased in terms of pseudomeasures. (Kahane omits the proof but says that it follows from the result we quoted as Theorem 2.3.) Kahane then remarks (using a different terminology) that the possibility of balayage for $(\Lambda, E)$ leads to results, such as the one of his referred to after our Theorem 6.3, on E's being contained in the zero sets of certain trigonometric sets. Indeed, in proving that result [6, p. 160], Kahane constructed BAS sets. Our construction of BAS sets seems less involved than Kahane's. His method, however, leads to results on both the possibility and impossibility of balayage with $\Lambda$ sparse but not arbitrarily sparse.

\section{BIBLIOGRAPHY}

1. W. G. Bade and P. C. Curtis, Embedding theorems for commutative Banach algebras, Pacific J. Math. 18 (1966), 391-409. MR 34 \# 1878.

2. A. Beurling, On balayage of measures in Fourier transforms, Notes from a seminar at the Institute for Advanced Study, Princeton, N.J., 1959-60 (unpublished).

3. - Local harmonic analysis with some applications to differential operators, Annual Science Conference Proceedings, vol. I: 1962-1964, Belfer Graduate School of Science, Academic Press, New York, 1966, pp. 109-125.

4. E. Hewitt and K. A. Ross, Abstract harmonic analysis. I, II, Grundlehren math Wiss., Bände 115, 152, Springer-Verlag, Berlin and New York, 1963, 1970. MR 28 \# 158; 41 \# 7378.

5. J.-P. Kahane, Séries de Fourier absolument convergentes, Ergebnisse der Mathematik und ihrer Grenzgebiete, Band 50, Springer-Verlag, Berlin and New York, 1970. MR 43 \#801.

6. J.-P. Kahane and R. Salem, Ensembles parfaits et séries trigonométriques, Actualités Sci. Indust., No. 1301, Hermann, Paris, 1963. MR 28 \#3279.

7. H. J. Landau, Necessary density conditions for sampling and interpolation of certain entire functions, Acta Math. 117 (1967), 37-52. MR 36 \#564.

8. Y. Meyer, Algebraic numbers and harmonic analysis, North-Holland Math. Library, no. 2, North-Holland, Amsterdam, 1972.

9. H. Reiter, Classical harmonic analysis and locally compact groups, Oxford Math. Monographs, Clarendon Press, Oxford, London, 1968. MR 46 \#5933. 1973.

10. G. S. Shapiro, Some aspects of balayage of Fourier transforms, Dissertation, Harvard Univ.,

11. N. Varopoulos, Sets of multiplicity in locally compact abelian groups, Ann. Inst. Fourier (Grenoble) 16 (1966), fasc. 2, 123-158. MR 35 \#3379.

Department of Mathematics, Brooklyn College (CUNY), Brooklyn, New York 11210 